\title{
The orbitopterygoid corridor as a deep keyhole for endoscopic access to the paranasal sinuses and clivus
}

\author{
*Kenichi Oyama, MD, PhD, ${ }^{1}$ Kentaro Watanabe, MD, ${ }^{1}$ Shunya Hanakita, MD, ${ }^{1}$ \\ Pierre-Olivier Champagne, MD, ${ }^{1}$ Thibault Passeri, MD, ${ }^{1}$ Eduard H. Voormolen, MD, PhD, ${ }^{1}$ \\ Anne Laure Bernat, MD, ${ }^{1}$ Nicolas Penet, MD, ${ }^{1}$ Takanori Fukushima, MD, ${ }^{2}$ and \\ Sébastien Froelich, MD ${ }^{1}$
}

1Department of Neurosurgery, Lariboisière Hospital, University of Paris Diderot, Paris, France, and 2Department of Neurological Surgery, Duke University, Durham, North Carolina

OBJECTIVE The anteromedial triangle (AMT) is the triangle formed by the ophthalmic (V1) and maxillary (V2) nerves. Opening of this bony space offers a limited access to the sphenoid sinus (SphS). This study aims to demonstrate the utility of the orbitopterygopalatine corridor (OPC), obtained by enlarging the AMT and transposing the contents of the pterygopalatine fossa (PPF) and V2, as an entrance to the SphS, maxillary sinus (MaxS), and nasal cavity.

METHODS Five formalin-injected cadaveric specimens were used for this study (10 approaches). A classic pterional approach was performed. An OPC was created through the inferior orbital fissure, between the orbit and the PPF, by transposing the PPF inferiorly. The extent of the OPC was measured using neuronavigation and manual measurements. Two illustrative cases using the OPC to access skull base tumors are presented in the body of the article.

RESULTS Via the OPC, the SphS, MaxS, ethmoid sinus (EthS), and nasal cavity could be accessed. The use of endoscopic assistance through the OPC achieved better visualization of the EthS, SphS, MaxS, clivus, and nasal cavity. A significant gain in the area of exposure could be achieved using the OPC compared to the AMT (22.4 mm² vs 504.1 $\left.\mathrm{mm}^{2}\right)$.

CONCLUSIONS Opening of the AMT and transposition of $\mathrm{V} 2$ and the contents of the PPF creates the OPC, a potentially useful deep keyhole to access the paranasal sinuses and clival region through a middle fossa approach. It is a valuable alternative approach to reach deep-seated skull base lesions infiltrating the cavernous sinus and middle cranial fossa and extending into the paranasal sinus.

https://thejns.org/doi/abs/10.3171/2020.3.JNS2022

KEYWORDS anteromedial triangle; endoscopic assistance; paranasal sinuses; pterygopalatine fossa; skull base

$\mathrm{T}$ HE anteromedial triangle (AMT), first described by Mullan, ${ }^{1}$ is defined by the lower margin of the ophthalmic nerve (V1), the upper margin of the maxillary nerve (V2), and a line connecting the point where V1 passes through the superior orbital fissure (SOF) and V2 passes through the foramen rotundum (FR) (Fig. 1). The superior ophthalmic vein exits the orbit through the SOF and passes the AMT posteriorly and inferiorly to V1, explaining why Mullan used this triangle to treat carotid-cavernous sinus fistulas. ${ }^{1}$ The AMT is one of the various anatomical triangles surrounding the cavernous sinus. ${ }^{2,3}$ Its surgical usefulness as an entry point into the sphenoid sinus ( $\mathrm{SphS}$ ) through the middle fossa has been described. It remains, however, on its own a limited corridor. ${ }^{4}$

In this study, we describe an expansion of the AMT corridor to obtain a wider surgical corridor, naming it the orbitopterygopalatine corridor (OPC). The surgical access to the nasal cavity, paranasal sinuses, and clivus through the OPC was first evaluated using cadaveric specimens.

ABBREVIATIONS AMT = anteromedial triangle; EEA = endoscopic endonasal approach; EthS = ethmoid sinus; FR = foramen rotundum; ICA = internal carotid artery; IOF = inferior orbital fissure; MaxS = maxillary sinus; $\mathrm{OM}=$ orbital muscle of Müller; $\mathrm{OPC}=$ orbitopterygopalatine corridor; $\mathrm{PPF}=$ pterygopalatine fossa; $\mathrm{SOF}=$ superior orbital fissure; $\mathrm{SphS}=$ sphenoid sinus.

SUBMITTED January 3, 2020. ACCEPTED March 31, 2020.

INCLUDE WHEN CITING Published online June 12, 2020; DOI: 10.3171/2020.3.JNS2022.

${ }^{*}$ K.O. and K.W. contributed equally to this work. 

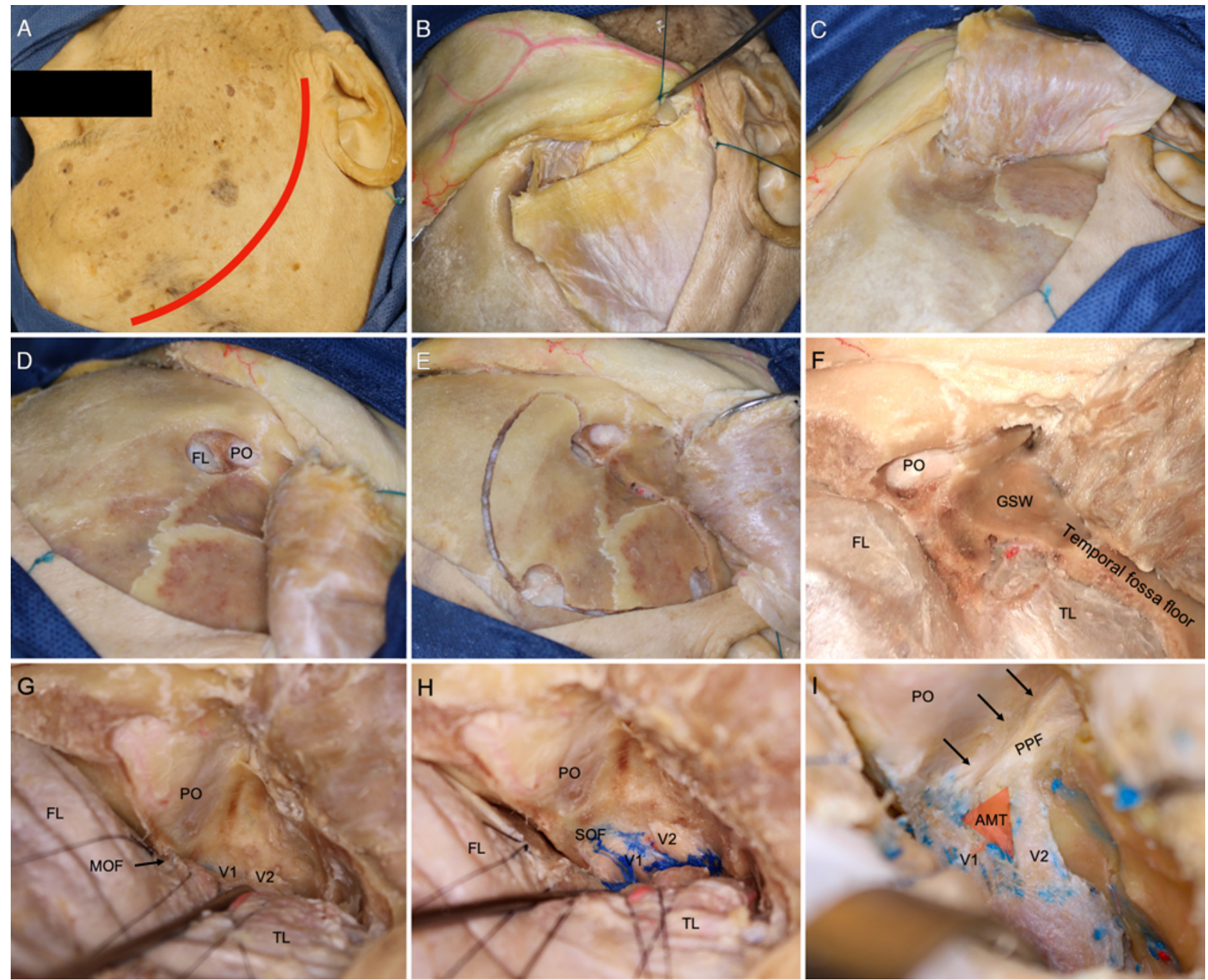

FIG. 1. Stepwise exposure of the AMT. A: Skin incision (red line) for the frontotemporal craniotomy starting in front of the tragus. B: An interfascial dissection is performed. C: The temporalis muscle is elevated using a retrograde dissection technique and transposed inferoposteriorly. D and E: After creating the MacCarty keyhole to identify the periorbita (PO) and the dura of the frontal lobe (FL), a frontotemporal craniotomy is performed. F: The greater (GSW) and lesser wing of the sphenoid bone and the temporal fossa floor are fully drilled. TL = temporal lobe. $\mathbf{G}$ and $\mathrm{H}$ : The lateral wall of the PO is resected. After cutting the meningoorbital fold (MOF), the dura of TL is further peeled to expose V1 and V2. I: The PPF and AMT (red triangle) are exposed, and the bone that forms the lateral margin of the IOF is removed. The print left behind once this bone is removed appears as a sulcus, which identifies the level of the IOF and the position of the OM (arrows). Figure is available in color online only.

We also present clinical cases to illustrate potential applications of this technique.

\section{Methods}

The study received approval from our institutional review board. Five formalin-fixed cadaveric heads injected with colored neoprene latex in accordance with previously described techniques ${ }^{5}$ were used for cadaveric dissections. A microscope (OPMI pico; Carl Zeiss, Inc.) and an endoscope (Karl Storz GmbH) were used for dissection assistance and photography. For endoscopic exploration, rigid 4-mm-diameter endoscopes that were $18 \mathrm{~cm}$ in length with $0^{\circ}$ and $30^{\circ}$ lenses (Karl Storz $\mathrm{GmbH}$ ) were used. Bone resection was performed with a high-speed drill (Midas
Rex; Medtronic), and intraoperative images were recorded and stored with the Aida system (Karl Storz GmbH). Measurement and evaluation of surgical trajectories were performed using a CT scan and a neuronavigation system (Stealth; Medtronic).

\section{Dissection Technique and Surgical Anatomy}

A standard frontotemporal craniotomy is first performed with the head fixed in a Mayfield head holder (Integra LifeScience) rotated $30^{\circ}$ to the contralateral side, with $5^{\circ}$ of extension. The pterion and the lesser and greater sphenoid wings are drilled, allowing opening of the SOF and removal of the lateral wall of the orbit anteriorly, exposing the periorbita (Fig. 1). During this step, the lateral 
rectus spine located on the orbital surface of the greater sphenoid wing is identified and removed. The temporal and frontal fossa dura is elevated, and the FR is exposed. The dura propria is then peeled from the anterior aspect of the cavernous sinus, exposing cranial nerves III and IV and V1 and V2 (Fig. 1). The greater wing and anterior aspect of the temporal fossa floor are further drilled, and the FR is unroofed. The pterygopalatine fossa (PPF) located anterior to FR (Fig. 1) is exposed, and the periosteum covering the PPF is left intact.

Once the PPF is exposed, the remaining bone between the orbit and the PPF that forms the lateral margin of the inferior orbital fissure (IOF) anterior to the maxillary strut is removed. Removing this bone with a rongeur most often disrupts the periosteum. This tight adherence is due to the attachment of the fibers of the orbital muscle of Müller $(\mathrm{OM})$. The print left behind once this bone is removed appears as a sulcus, which identifies the level of the IOF and the position of the OM (Fig. 1).

The maxillary strut is drilled and the SphS is entered. The bone lateral to the FR can be further drilled to obtain more inferior mobilization of V2, which enlarges the AMT (Fig. 2).

To expand the ATM corridor anteriorly, the periosteum is opened between the orbit and the PPF at the level of the sulcus left behind by the removal of the lateral margin of the IOF. The OM is then identified, having a brownish color easily distinguished from the yellow orbital extraconal fat above it and the PPF fat below (Fig. 3). Above the maxillary sinus (MaxS), below the level of the OM and the IOF, the posterior extension of the PPF through the SOF is identified. It is sectioned above the maxillary strut to allow the mobilization of the PPF and V2 inferiorly, thus enlarging the OPC (Fig. 3).

Below the level of the OM and IOF, the extracranial V2 is identified, surrounded by fat tissue (Fig. 4). V2 is followed anteriorly until it reaches the infraorbital groove. The zygomatic nerve, a branch of V2 crossing the IOF and $\mathrm{OM}$ and running along the lateral orbital wall, is identified (Fig. 4).

Above the level of the OM and IOF, the periorbita can be elevated from the inferior wall of the orbit, and the fat of the inferior extraconal compartment can be safely removed to increase the surgical corridor (Fig. 4).

The OM is then removed, and the medial margin of the IOF is exposed. It is formed anteriorly by the maxilla and posteriorly by the orbital process. The opening of the $\mathrm{SphS}$ made at the level of the maxillary strut is extended anteriorly by drilling the medial margin of the IOF and the inferior wall of the orbit, creating a wide opening of the SphS. Depending on its location, the zygomatic nerve might be sacrificed, especially when it is close to the anterior margin of the maxillary strut.

More anteriorly, the sphenopalatine foramen, sphenopalatine artery, and palatine nerve, a branch from the maxillary nerve, are identified (Fig. 4).

The posterior wall of the MaxS, located anteriorly below V2 before it enters the infraorbital groove, can be exposed (Fig. 4). This very thin wall of bone can be easily broken and enlarged to obtain a wide window for endoscopic exploration (Fig. 2).

\section{Measurements}

The OPC was exposed using the aforementioned steps on each side of the 5 cadaveric specimens (10 approaches total). The following measurements were performed on each side for all the heads at various steps of exposure: distance from the V1-V2 angle and SOF, distance from the V1-V2 angle and anterior end of the FR, distance between the SOF and the anterior end of the FR, distance between the anterior border of the maxillary strut and zygomatic nerve, distance between the zygomatic nerve and IOF, and the maximal length and width of the OPC (Fig. 5). To compare the added value of the OPC over the traditional AMT exposure, the surfaces of both the AMT and OPC were calculated from the measurements. The AMT surface was calculated using Heron's formula $[p(p-a)(p$ $-b)(p-c)]^{1 / 2}$ for triangles, and the OPC surface was calculated using the ellipsoid method $(\mathrm{r} \times \mathrm{R} \times \pi)$.

\section{Results}

In all 10 sides of the specimens, the OPC was successfully exposed using the aforementioned surgical steps. The mean length from the top of the angle between V1 and V2 to the SOF was $10.6 \pm 3.0 \mathrm{~mm}$, from the top of the angle between V1 and V2 to the FR was $9.0 \pm 1.7 \mathrm{~mm}$, and from the SOF to the FR was $5.5 \pm 1.2 \mathrm{~mm}$. The mean distance from the most anterior point of the maxillary strut to the origin of the zygomatic nerve was $4.6 \pm 1.9 \mathrm{~mm}$, from the origin of the zygomatic nerve to the distal point of the IOF was $21.0 \pm 3.4 \mathrm{~mm}$, and from the top of the angle between $\mathrm{V} 1$ and $\mathrm{V} 2$ to the distal point of the IOF was 25.5 $\pm 4.5 \mathrm{~mm}$. The mean size of the OPC obtained with this technique was $14.2 \pm 1.9 \mathrm{~mm}$ in width and $12.3 \pm 0.9 \mathrm{~mm}$ in height. The mean surface of the AMT was $22.4 \mathrm{~mm}^{2}$, and the mean surface of the OPC was $504.1 \mathrm{~mm}^{2}$. These findings are summarized in Table 1.

\section{Endoscopic Visualization Through the V1-V2 Corridor}

After opening the SphS, the MaxS, and the ethmoid sinus (EthS), an endoscope was introduced and anatomical landmarks were identified (Fig. 2). The endoscopic perspective via the OPC provided a wide surgical view into the SphS, including visualization of the intrasphenoidal septum, the sella turcica, clival depression, and the contralateral internal carotid artery (ICA) (Fig. 2). The midclivus was further drilled to expose the clival dura using a curved high-speed drill.

The various walls of the MaxS were explored using angled scopes (Fig. 2). Following the sphenopalatine artery medially, the sphenopalatine foramen was identified. The anterior margin of the sphenopalatine foramen and the medial wall of the MaxS were removed in order to enter the nasal cavity immediately above the root of the inferior turbinate (Fig. 2). Using angle scopes, the medial meatus was explored.

\section{Illustrative Cases \\ Case 1}

A 41-year-old man presented with exophthalmos secondary to a sphenoorbital meningioma that extended into 


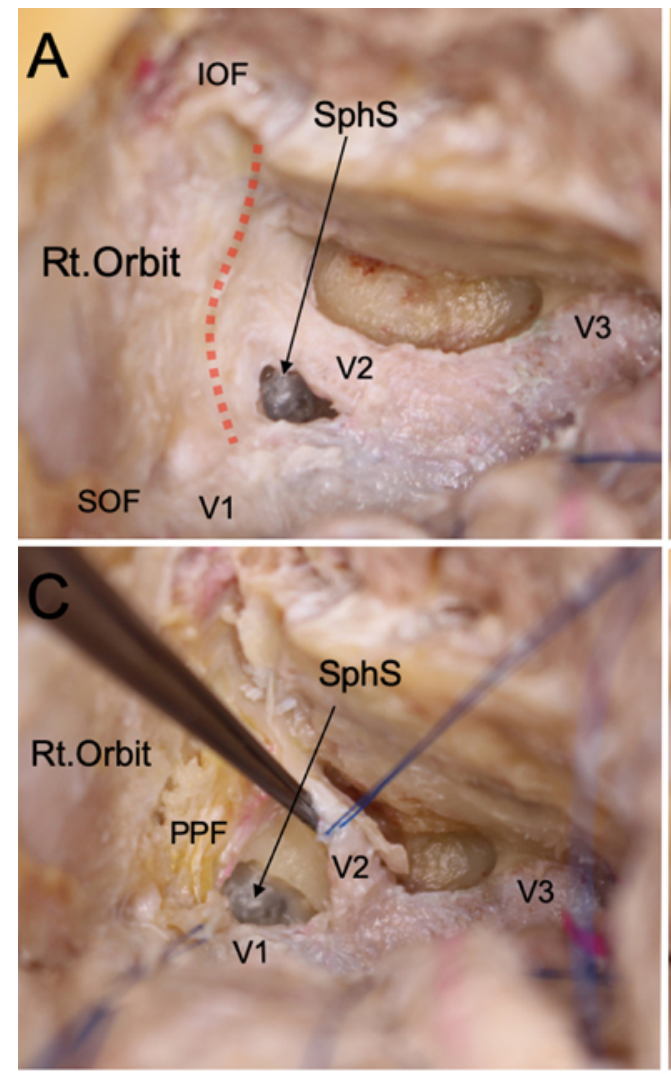

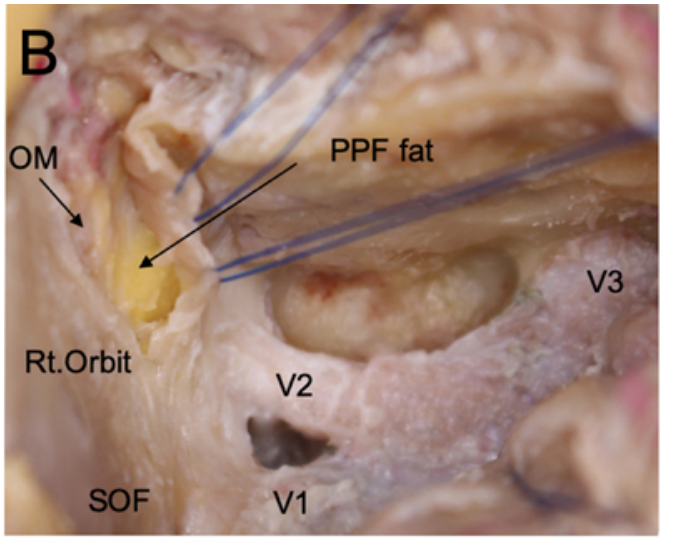

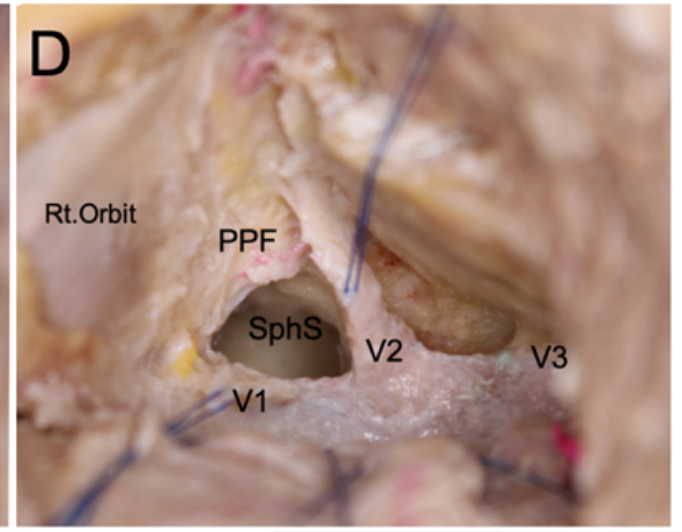

FIG. 2. Opening the SphS through the enlarged AMT. A: The maxillary strut is drilled to enter the SphS. The bone lateral to the FR and V2 is drilled in order to allow the lateral mobilization of V2. The dotted line indicates the level of the IOF. V3 = mandibular nerve. $\mathrm{B}$ : The periosteum covering the PPF is opened, and V2 is mobilized laterally. $\mathbf{C}$ and D: The opening of the SphS is enlarged. E: View of OPC prior to insertion of the endoscope. F: Endoscopic view through the OPC showing the SphS, MaxS, and EthS. G: Endoscopic view of the SphS with a $30^{\circ}$ scope. The posterior wall of the SphS has been drilled, showing the pituitary, right and left paraclival ICA, and clival dura. The contralateral vidian canal has been unroofed. H: Endoscopic view of the medial wall and superior wall of the MaxS using a $30^{\circ}$ scope. I: Endoscopic view of the middle meatus after removal of the medial wall of the MaxS. FIG. 2. (continued) $\rightarrow$

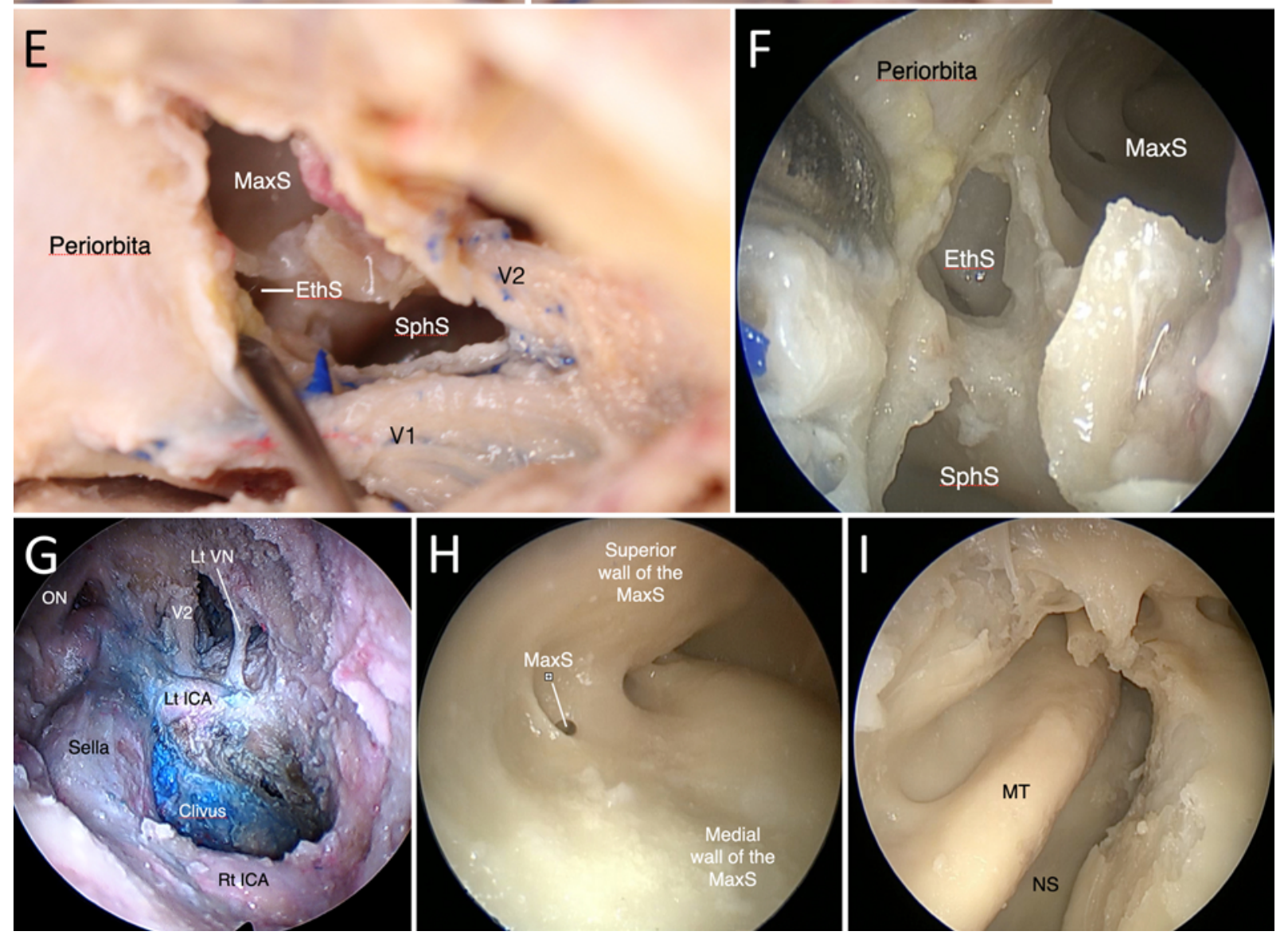


Oyama et al.

FIG. 2. $E S=$ EthS; $M S=$ MaxS $; M S O=$ MaxS ostium; $M T=$ middle turbinate; $N S=$ nasal septum; $O M=$ orbital muscle; ON = optic nerve; $S S=S p h S ;$ $\mathrm{VN}=$ vidian nerve. Figure is available in color online only.

the anterior clinoid process and the SphS (Fig. 6). Following a pterional craniotomy and peeling of the lateral wall of the cavernous sinus, an extradural anterior clinoidectomy was first performed. The intradural part of the tumor was resected, together with the invaded dura mater and periorbita. After a watertight closure of the dural defect using a patch of pericranium, the FR was unroofed and the PPF was exposed. Tumor seeding along the V2 nerve sheath was removed. The MaxS was drilled and the SphS was opened. The OPC was then created. The posterior extension of the PPF through the SOF above the MaxS was sectioned, and the PPF was transposed inferiorly. The OM was removed and the perirobita was elevated from the inferior wall of the orbit. The medial aspect of the IOF was drilled and the $\mathrm{SphS}$ was entered. The tumor in the $\mathrm{SphS}$ was visualized and resected in most parts under microscopic view (Fig. 7).
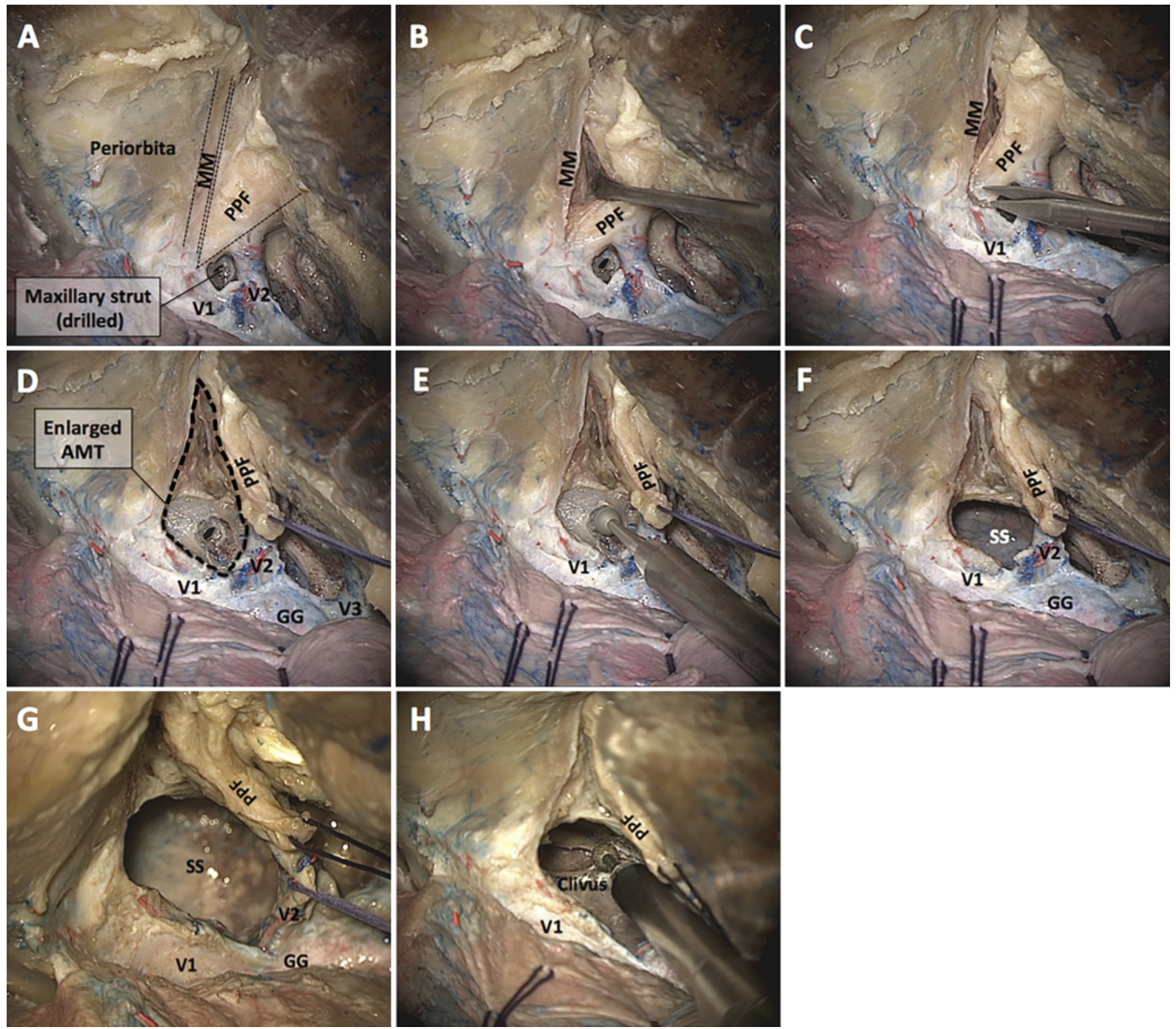

FIG. 3. Inferior transposition of the PPF and stepwise exposure of the OPC. A: The level of the OM (MM) and IOF is indicated. B: The periosteum is opened between the orbit and PPF. C: Above the MaxS, below the level of the OM and IOF, the posterior extension of the PPF through the SOF is identified and sectioned. D: The PPF is retracted inferiorly, creating a wide corridor between the orbit and PPF. E: The maxillary strut and the medial aspect of the IOF are drilled in order to enter into the SphS. F: The SphS is widely opened. G: The OPC is extended anteriorly. H: Through the OPC and the SphS, the clivus is drilled. GG = gasserian ganglion. Figure is available in color online only. 

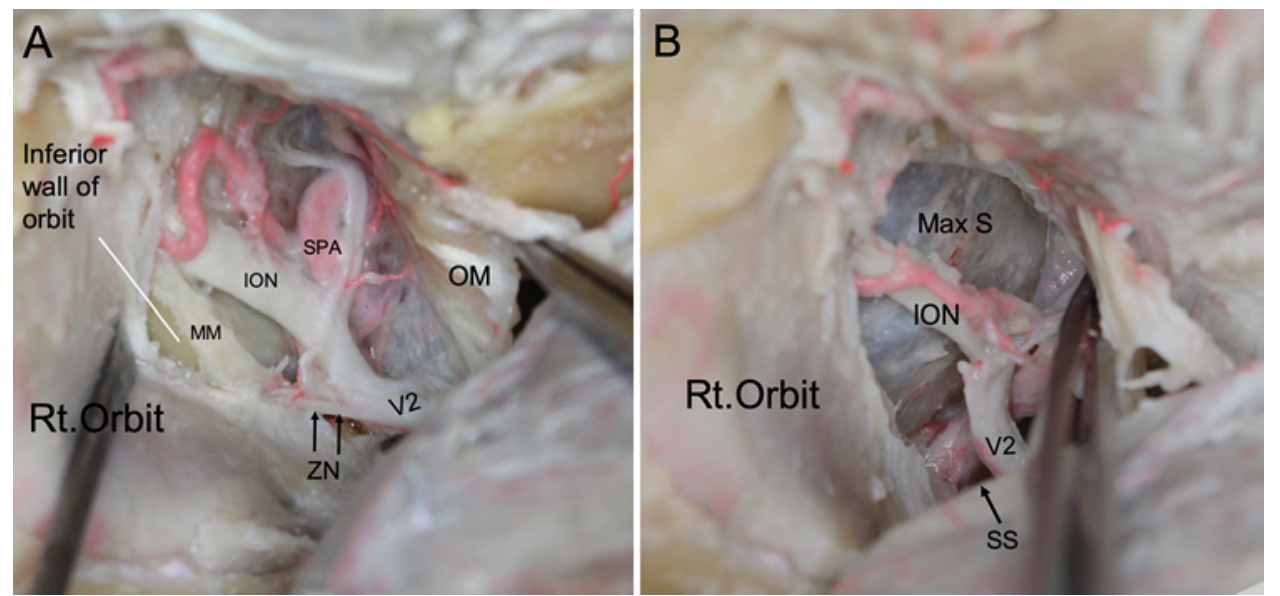

FIG. 4. Anterior part of the OPC. Exposure of the MaxS. A: After opening the PPF and removing the fat in the PPF, the inferior orbital nerve (ION), the sphenopalatine artery (SPA), and other branches of the maxillary artery come into view. The OM indicates the level of the IOF and separates the PPF from the orbit. The zygomatic nerve (ZN) crosses the IOF. B: The OM and the medial margin of the IOF have been removed. The mucosa of the posterior wall of the MaxS is exposed. Figure is available in color online only.
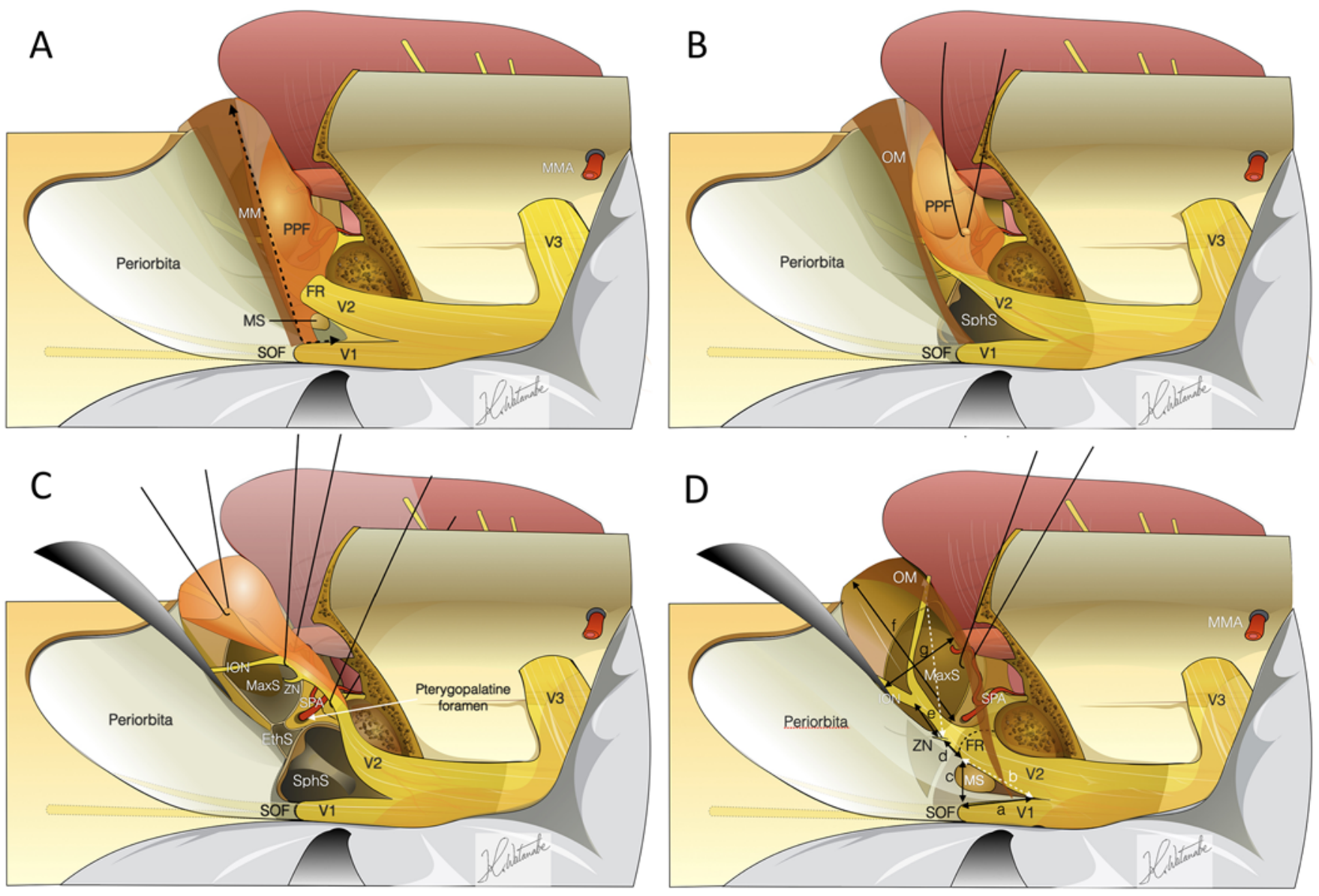

FIG. 5. Drawing of the surgical corridor to the SphS and MaxS. A: The dotted line indicates the level of the IOF and OM (MM). MMA = middle meningeal artery. B: Transposition of the PPF and maxillary nerve provides significant access to the SphS. C: Further transposition of the PPF and maxillary nerve gives access to the MaxS and sphenopalatine foramen. D: Landmarks and measurements used to evaluate the exposure. Each distance was measured as follows: distance between the top of the V1-V2 angle and the SOF (a); distance between the top of the V1-V2 angle and the anterior end of FR (b); distance between the SOF and FR (c); distance between the anterior border of the maxillary strut and zygomatic nerve (ZN) (d); distance between the ZN and the infraorbital nerve (e); the maximal length of the OPC (f); and the maximal width of the OPC (g). Copyright Kentaro Watanabe. Published with permission. Figure is available in color online only. 
Oyama et al.

TABLE 1. Summary of measurements in 5 cadaveric heads (10 sides)

\begin{tabular}{lc}
\hline \multicolumn{1}{c}{ Landmark } & Mean \\
\hline AMT surface & $22.4 \mathrm{~mm}^{2}$ \\
\hline V1-V2 angle-SOF & $10.6 \mathrm{~mm}$ \\
\hline V1-V2 angle-FR & $9.0 \mathrm{~mm}$ \\
\hline V1-V2 angle-IOF & $25.5 \mathrm{~mm}$ \\
\hline SOF-FR & $5.5 \mathrm{~mm}$ \\
\hline MSt-ZN & $4.6 \mathrm{~mm}$ \\
\hline ZN-IOF & $21.0 \mathrm{~mm}$ \\
\hline Major diameter of OPC & $14.2 \mathrm{~mm}$ \\
\hline Minor axis of OPC & $12.3 \mathrm{~mm}$ \\
\hline OPC surface & $504.1 \mathrm{~mm}$ \\
\hline
\end{tabular}

$\mathrm{MSt}=$ maxillary strut $\mathrm{ZN}=$ zygomatic nerve.

A $30^{\circ}$ angled endoscope was used for inspection of any tumor remnant in the SphS. The closure of the opened SphS was performed with temporal muscle graft that was sealed with a tissue glue-coated collagen sponge (TachoSil; Baxter) and fibrin glue. The histopathology was grade I meningothelial meningioma. The symptom of exophthalmos immediately improved, and the postoperative course was uneventful. V2 function including corneal sensation was normal postoperatively, and although lacrimation was not formally tested, no subjective eye dryness was noted during the postoperative course. Postoperative imaging confirmed gross-total resection of the tumor (Fig. 6).

\section{Case 2}

A 52-year-old woman presented with trigeminal neuralgia and hypoesthesia in the territory of the second division of the trigeminal nerve. MRI indicated a cavernous meningioma extending toward the temporal fossa and into the sphenopalatine fossa and SphS. Two MR images at the 6-month interval indicated significant tumor growth. A decision was made for the patient to undergo surgery, with the objective of resecting the extracavernous part of the tumor and leaving the remaining part in the cavernous sinus for radiosurgery. A frontotemporal approach was performed. The dura was peeled from the cavernous sinus. The infiltrated dura and the intradural temporal extension of the tumor were removed, and the dural defect was closed with a patch of pericranium. Then the greater sphenoid wing and the anterior aspect of the temporal fossa floor were drilled. The FR was unroofed. Tumor extending into the sphenopalatine fossa was removed. After establishing the OPC, the SphS was entered and tumor protruding into
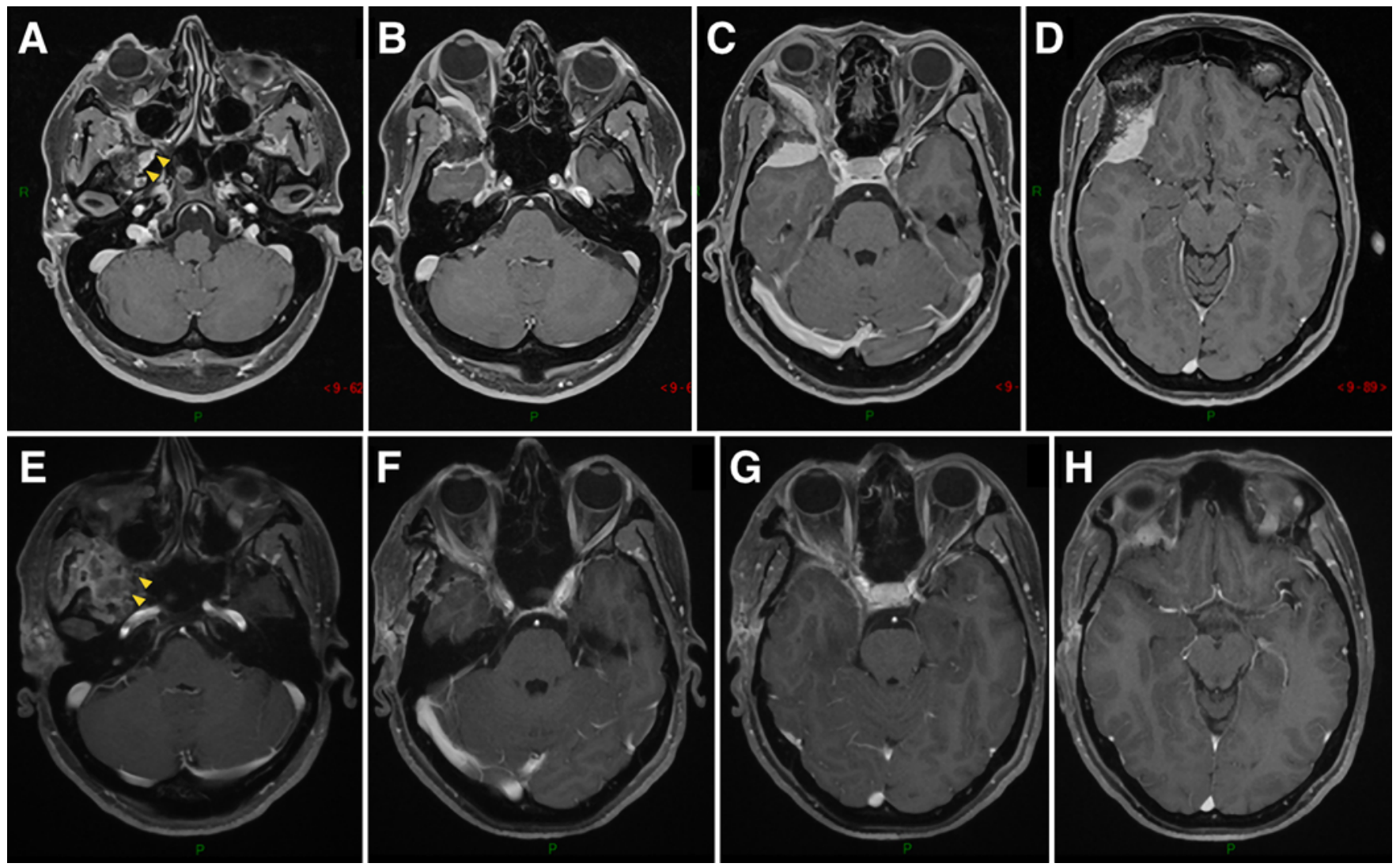

FIG. 6. Imaging of illustrative case 1. A-D: Preoperative axial MR images showing a contrast-enhancing lesion in the sphenoorbital area extending into the sphenopalatine fossa (yellow arrowheads). E-H: Postoperative axial MR images demonstrating gross-total resection of the tumor. Figure is available in color online only. 


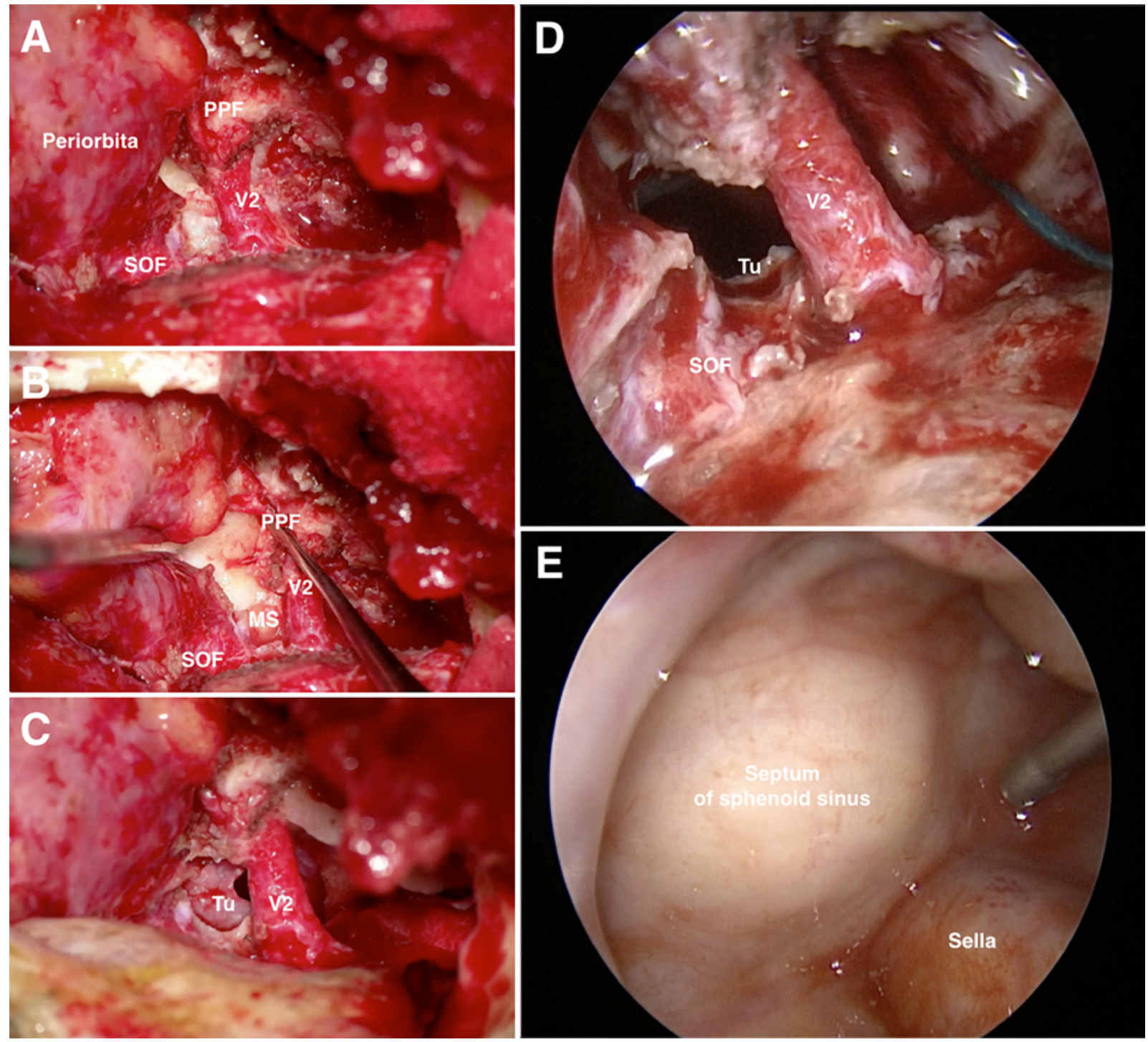

FIG. 7. Intraoperative images of case 1. A: The SOF, PPF, and V2 are exposed. B: The maxillary strut (MS) is seen between V2 and the SOF, and the PPF is transposed. C: Tumor (Tu) is seen in the SphS. D: Endoscopic intraoperative view showing the enlarged AMT and OPC. E: Endoscopic view in the SphS. Figure is available in color online only.

the sinus was found. The SphS was also entered lateral to V2. The tumor in the SphS was resected under endoscopic visualization. This approach allowed for removal of tumor present in both the intradural space and paranasal sinuses. Tumor in the cavernous sinus was left voluntarily to preserve function. After resection of the tumor, the opened SphS was closed in the same fashion described in case 1. The patient's postoperative course was uneventful. V2 function was not worsened postoperatively, and although lacrimation was not formally tested, no subjective eye dryness was noted during the postoperative course. The histopathology was a grade I meningioma with a Ki-67 index at $10 \%$. Because of the rapid growth of the tumor preoperatively, the patient underwent radiosurgery 3 months after surgery. The patient had no sign of progression 16 months after radiosurgery. An operative video of case 2 is shown (Video 1).
VIDEO 1. Operative video of case 2 demonstrating the major steps of the approach. Copyright Sébastien Froelich. Published with permission. Click here to view.

\section{Discussion}

In this study, we describe the OPC, a transcranial surgical corridor to the paranasal sinuses, nasal cavity, and clivus, that was created by enlarging the AMT (V1-V2 triangle). Endoscopic visualization through the OPC provides a wide surgical view of the SphS, MaxS, and the clival region.

\section{Indications of the Approach}

Intracranial lesions with significant extensions outside the cranium toward the PPF and paranasal sinuses represent the main indication for this approach (meningiomas, 
chondrosarcomas, and chordomas). Of note, these tumors can sometimes enlarge the AMT and FR, easing the creation of the orbitopterygoid corridor. Various transcranial approaches have been proposed to gain access to this region. ${ }^{3,6-11}$ More recently, endoscopic endonasal approaches (EEAs) have become an important alternative to traditional transcranial approaches, especially for ventral skull base lesions. ${ }^{12,13}$ The majority of lesions seated in paranasal sinuses and the pterygopalatine region can thus be treated via an EEA. ${ }^{12-21}$ Although some access to the AMT can be achieved using an EEA, ${ }^{22,23}$ these tumors sometimes have significant intradural extensions into the cavernous sinus, lateral to the cavernous ICA and cranial nerves, or into the temporal fossa, making them not easily amenable to resection via an endonasal corridor because of risk of injury to neurovascular complexes. ${ }^{24,25}$ In addition to the significant risk of a CSF leak, a more extensive EEA in the coronal plane can also increase the risk of endonasal morbidity such as chronic MaxS, crusting, runny nose, and loss of smell. ${ }^{26}$

By using the OPC as a gateway to the nasal cavity, SphS, MaxS, and clivus, our technique combines the advantages of both transcranial and endonasal approaches. The presented approach makes it possible to manipulate critical neurovascular structures while avoiding sinonasal trauma (advantages of transcranial approach) and provides a useful surgical corridor to the sinonasal cavities and clivus (advantages of EEA).

One of the limitations of the surgical exposure is the clival bone abutting the paraclival ICA on the ipsilateral side. Also, although the entire SphS can be visualized and reached via this approach using angled endoscopes and curved instruments, access to the contralateral side remained limited. If the lesion extends to the contralateral side, other surgical approaches or a subsequent surgical stage should be considered. For example, an EEA could be added in order to gain better access to the nasal sinuses, and it also offers the possibility of adding a pedicled flap (such as the nasoseptal flap) to the reconstruction. If the lesion extends laterally in the pterygoid plate and infratemporal fossa, one option is also to add the corridor obtained by removing the bony structure at $\mathrm{V} 2-\mathrm{V} 3$ corridor.

\section{Closure}

Using this technique, the PPF, nasal cavity, EthS, SphS, and MaxS are opened, creating a connection between the paranasal sinuses and intracranial spaces that needs to be carefully closed in order to avoid a CSF leak. To potentially reduce the risk of infection, the intradural part of the tumor is usually resected first, then the defect of dura mater is closed before opening the paranasal sinuses, using pericranium that is harvested at the beginning of the craniotomy. The OPC is then closed using a fat graft or a piece of temporalis muscle, depending on the size of the opening. As another option, the vascularized temporoparietal fascia flap, as used in EEAs, ${ }^{27}$ can be also used in cases of large defects and a high risk of CSF leak.

\section{Potential Morbidity of the Approach}

In addition to the potential risk of a CSF leak and meningitis from opening the paranasal sinuses, opening of the nasal cavity, EthS, MaxS, and SphS may cause other morbidities, such as chronic MaxS and crusting. The zygomatic nerve carries the parasympathetic fibers to the frontal branch to the trigeminal nerve, which innervates the lacrimal gland and conjunctiva.$^{28}$ Sacrifice of the zygomatic nerve is usually necessary to increase the size of the OPC. Its sacrifice can lead to a dry eye, which should be seriously considered, especially if the surgical manipulation increases the risk of V1 injury and corneal anesthesia. Manipulations of V2 to increase the working space can also cause facial dysesthesia postoperatively. Gentle manipulation and meticulous hemostasis without monopolar or bipolar coagulation close to the nerve are preferred. It is also important to be mindful that the endoscopic keyhole access given via the orbitopterygoid corridor puts proximal structures (mostly V1, V2, and zygomatic nerve) at risk from unseen manipulations once the endoscope is brought in.

\section{Limitations of the Study}

This study is limited by its nature as a cadaveric study. The dissections were performed on normal anatomy in a controlled setting, not taking into account the variability and unpredictability of live surgery. This is partially compensated by the two illustrative cases, which exemplify the feasibility and potential utility of the approach.

\section{Conclusions}

We describe the surgical technique through the OPC obtained by enlarging the AMT. The OPC can provide a useful surgical gateway to the nasal cavity, SphS, MaxS, and the clivus. Combined use of the microscope and endoscope enables improved visualization and illumination for tumor resection through this corridor. This novel approach can be an option for pathologies in the sphenoorbital region with extension to the paranasal sinuses, especially in cases needing meticulous intradural manipulation.

\section{References}

1. Mullan S. Treatment of carotid-cavernous fistulas by cavernous sinus occlusion. J Neurosurg. 1979;50(2):131-144.

2. Rhoton AL Jr. The cavernous sinus, the cavernous venous plexus, and the carotid collar. Neurosurgery. 2002;51(4) (suppl):S375-S410.

3. Sekhar LN, Møller AR. Operative management of tumors involving the cavernous sinus. J Neurosurg. 1986;64(6):879889.

4. Sincoff EH, Liu JK, Matsen L, et al. A novel treatment approach to cholesterol granulomas. Technical note. J Neurosurg. 2007;107(2):446-450.

5. Sanan A, Abdel Aziz KM, Janjua RM, et al. Colored silicone injection for use in neurosurgical dissections: anatomic technical note. Neurosurgery. 1999;45(5):1267-1274.

6. Dolenc VV. Frontotemporal epidural approach to trigeminal neurinomas. Acta Neurochir (Wien). 1994;130(1-4):55-65.

7. Fisch U. Infratemporal fossa approach to tumours of the temporal bone and base of the skull. J Laryngol Otol. 1978;92(11):949-967.

8. Hakuba A, Tanaka K, Suzuki T, Nishimura S. A combined orbitozygomatic infratemporal epidural and subdural approach for lesions involving the entire cavernous sinus. $J$ Neurosurg. 1989;71(5 Pt 1):699-704. 
9. Ohue S, Fukushima T, Kumon Y, et al. Preauricular transzygomatic anterior infratemporal fossa approach for tumors in or around infratemporal fossa lesions. Neurosurg Rev. 2012;35(4):583-592.

10. Parkinson D. A surgical approach to the cavernous portion of the carotid artery. Anatomical studies and case report. $J$ Neurosurg. 1965;23(5):474-483.

11. Yoshida K, Kawase T. Trigeminal neurinomas extending into multiple fossae: surgical methods and review of the literature. J Neurosurg. 1999;91(2):202-211.

12. Cavallo LM, Cappabianca P, Galzio R, et al. Endoscopic transnasal approach to the cavernous sinus versus transcranial route: anatomic study. Neurosurgery. 2005;56(2) (suppl):379-389.

13. Cavallo LM, Messina A, Gardner P, et al. Extended endoscopic endonasal approach to the pterygopalatine fossa: anatomical study and clinical considerations. Neurosurg Focus. 2005;19(1):E5.

14. Chibbaro S, Cornelius JF, Froelich S, et al. Endoscopic endonasal approach in the management of skull base chordomasclinical experience on a large series, technique, outcome, and pitfalls. Neurosurg Rev. 2014;37(2):217-225.

15. de Notaris M, Cavallo LM, Prats-Galino A, et al. Endoscopic endonasal transclival approach and retrosigmoid approach to the clival and petroclival regions. Neurosurgery. 2009;65(6) (suppl):42-52.

16. Frank G, Sciarretta V, Calbucci F, et al. The endoscopic transnasal transsphenoidal approach for the treatment of cranial base chordomas and chondrosarcomas. Neurosurgery. 2006;59(1)(suppl 1):ONS50-ONS57.

17. Kassam A, Snyderman CH, Mintz A, et al. Expanded endonasal approach: the rostrocaudal axis. Part II. Posterior clinoids to the foramen magnum. Neurosurg Focus. 2005;19(1):E4.

18. Kassam AB, Gardner P, Snyderman C, et al. Expanded endonasal approach: fully endoscopic, completely transnasal approach to the middle third of the clivus, petrous bone, middle cranial fossa, and infratemporal fossa. Neurosurg Focus. 2005;19(1):E6.

19. Morera VA, Fernandez-Miranda JC, Prevedello DM, et al. "Far-medial" expanded endonasal approach to the inferior third of the clivus: the transcondylar and transjugular tubercle approaches. Neurosurgery. 2010;66(6)(suppl operative):211-220.

20. Oyama K, Ikezono T, Tahara S, et al. Petrous apex cholesterol granuloma treated via the endoscopic transsphenoidal approach. Acta Neurochir (Wien). 2007;149(3):299-302.

21. Stippler M, Gardner PA, Snyderman CH, et al. Endoscopic endonasal approach for clival chordomas. Neurosurgery. 2009;64(2):268-278.

22. Hanakita S, Chang WC, Watanabe K, et al. Endoscopic endonasal approach to the anteromedial temporal fossa and mobilization of the lateral wall of the cavernous sinus through the inferior orbital fissure and V1-V2 corridor: an anatomic study and clinical considerations. World Neurosurg. 2018;116:e169e178.
23. Komatsu F, Oda S, Shimoda M, et al. Endoscopic endonasal approach to the middle cranial fossa through the cavernous sinus triangles: anatomical considerations. Neurol Med Chir (Tokyo). 2014;54(12):1004-1008.

24. Thorp BD, Sreenath SB, Ebert CS, Zanation AM. Endoscopic skull base reconstruction: a review and clinical case series of 152 vascularized flaps used for surgical skull base defects in the setting of intraoperative cerebrospinal fluid leak. Neurosurg Focus. 2014;37(4):E4.

25. Zwagerman NT, Zenonos G, Lieber S, et al. Endoscopic transnasal skull base surgery: pushing the boundaries. $J$ Neurooncol. 2016;130(2):319-330.

26. McCoul ED, Bedrosian JC, Akselrod O, et al. Preservation of multidimensional quality of life after endoscopic pituitary adenoma resection. J Neurosurg. 2015;123(3):813-820.

27. Veyrat M, Verillaud B, Herman P, Bresson D. How I do it. The pedicled temporoparietal fascia flap for skull base reconstruction after endonasal endoscopic approaches. Acta Neurochir (Wien). 2016;158(12):2291-2294.

28. Martins C, Li X, Rhoton AL Jr. Role of the zygomaticofacial foramen in the orbitozygomatic craniotomy: anatomic report. Neurosurgery. 2003;53(1):168-173.

\section{Disclosures}

The authors report no conflict of interest concerning the materials or methods used in this study or the findings specified in this paper.

\section{Author Contributions}

Conception and design: Froelich, Fukushima. Acquisition of data: Oyama, Watanabe, Hanakita. Analysis and interpretation of data: Oyama, Watanabe, Hanakita. Drafting the article: Froelich, Oyama, Watanabe, Hanakita, Champagne. Critically revising the article: all authors. Reviewed submitted version of manuscript: all authors. Approved the final version of the manuscript on behalf of all authors: Froelich. Administrative/technical/material support: Froelich, Fukushima. Study supervision: Froelich, Fukushima.

\section{Supplemental Information Videos}

Video 1. https://vimeo.com/405384586.

\section{Correspondence}

Sébastien Froelich: Hôpital Lariboisière, Paris VII-Diderot University, Paris, France. sebastien.froelich@1rb.aphp.fr. 